\title{
Consejería nutricional incrementa el consumo de alimentos ricos en calcio, pero la ingesta se mantiene por debajo del requierimiento diario
}

\author{
Nutritional counseling increases \\ consumption of calcium-rich foods, \\ but mean intake remains below \\ the daily requirement
}

\begin{abstract}
Calcium (Ca) plays a crucial role in the regulation of metabolic processes, especially neuromuscular function and bone health. In Chile, calcium intake in women of childbearing age does not meet the dietary requirement. Objective: To determine whether standard and virtual nutritional counseling increases the dietary calcium intake in Chilean women of childbearing age. Subjects and Methods: Dietary calcium intake in 20 women (mean age 39 \pm 5 years) before and after a nutritional education intervention was compared. Nutritional counseling was provided to promote daily consumption of calcium-rich foods. Subsequently, a virtual space was provided to enhance standard counseling. On days 1, 30 and 62 a semi-quantitative food frequency questionnaire was completed by the women. Calcium intake significantly increased post intervention (ANOVA $F=4.43, P=$ 0.02). Dietary calcium intake was $308 \pm 116 \mathrm{mg} / \mathrm{d}$ at baseline, $354 \pm 138 \mathrm{mg} / \mathrm{d}$ at 30 days, and $412 \pm 188 \mathrm{mg} / \mathrm{d}$ after 62 days. Post intervention, 95\% of the participants did not meet the daily intake requirement for calcium; the average percent of adequate intake of calcium was $68 \pm 19 \%$. Conclusion: Nutritional counseling increased dietary calcium intake in women of reproductive age, but the increase was not enough to meet the daily calcium requirements.
\end{abstract}

Key words: Calcium, micronutrients, dietary intake, nutritional requirements, nutritional counseling.

\section{INTRODUCCIÓN}

El calcio es un mineral esencial que participa en la regulación de diversos procesos metabólicos, especialmente a nivel óseo y neuromuscular $(1,2)$. Este micronutriente juega un rol crucial en el desarrollo de huesos y dientes, en la conducción nerviosa, contracción muscular, ritmos cardíacos, función cardiovascular, entre otros $(1,3)$.

La ingesta dietaria de calcio es importante para mantener la salud ósea (4). La infancia y la adolescencia son los períodos de la vida más críticos para alcanzar la masa ósea máxima durante la edad adulta (5). La mujer empieza a perder masa ósea a partir de los 40 años, debido al proceso de resorción del hueso y a la carencia de estrógeno después de la menopausia,
Sheila Cerezo de Ríos $(1,2$,

Israel Ríos-Castillo $(1,3)$

Alex Brito O. (4)

Daniel López de Romaña $(4,5)$ Manuel Olivares G. (4,)

Fernando Pizarro A. (4.)

(1) Programa de Magíster en Nutrición y Alimentos. Instituto de Nutrición y Tecnología de los Alimentos (INTA), Universidad de Chile. Santiago, Chile. (2) Departamento de Nutrición, Caja de Seguro Social, Policlínica Santiago Barraza, La Chorrera, Panamá.

(3) Fundación para la Investigación Nutricional y Desarrollo Integral Oportuno, Ciudad de Panamá, Panamá. (4) Laboratorio de Micronutrientes, Instituto de Nutrición y Tecnología de los Alimentos (INTA) Universidad de Chile Santiago, Chile. (5) Micronutrient Initiative, Ottawa, Ontario, Canadá

Dirigir la correspondencia a: Nutricionista Sheila Cerezo de Ríos Caja de Seguro Social Policlínica Santiago Barraza Caja de Seguro Social, La Chorrera, Panamá Teléfonos: +507 253-3270, +507 69210271 E-mail: sheila.cerezo@hotmail.com

Este trabajo fue recibido el 14 de Febrero de 2014 y aceptado para ser publicado el 19 de Mayo de 2014.

aumentando el riesgo de osteoporosis $(6,7)$. Datos de la Organización Mundial de la Salud indican que la osteoporosis es más frecuente en las mujeres; afecta a más de 75 millones de personas en los Estados Unidos de América, Europa y Japón; y es la causa de 8,9 millones de fracturas anualmente en el mundo de las cuales 4,5 millones ocurren en las Américas y en Europa (8).

El Requerimiento Promedio Estimado (EAR, por sus siglas en inglés) de calcio es de 1000 mg/día para adultos jóvenes y mujeres en edad fértil (3). Sin embargo, se ha demostrado que el consumo de calcio en la región latinoamericana se encuentra por debajo de los requerimientos para la mayoría de los grupos etarios (9-12). En este sentido, la consejería 
nutricional ha demostrado aumentar el consumo de alimentos con alto contenido de este mineral $(13,14)$. Se ha reportado que intervenciones nutricionales por medio de herramientas virtuales han sido efectivas en promover la modificación de conductas alimentarias (15-17).

El año 2011 se llevó a cabo un estudio experimental para determinar el efecto de la administración oral de calcio sobre la absorción de hierro en mujeres chilenas en edad fértil (18). El siguiente análisis corresponde a un objetivo secundario predefinido de evaluación del efecto de la consejería nutricional presencial y virtual en la promoción de la ingesta dietaria de calcio en mujeres participantes en dicho estudio.

\section{SUJETOS Y MÉTODOS}

Estudio con diseño antes y después de una intervención de educación alimentaria y nutricional en 28 mujeres chilenas cuya edad media era $39 \pm 5$ años. Las mujeres fueron reclutadas de la comuna de Macul y La Florida del Sur Oriente de Santiago de Chile, un área de nivel socioeconómico bajo. El muestreo fue por conveniencia y utilizando la metodología en cadena, conocida como muestreo de bola de nieve. El estudio se realizó en el Laboratorio de Micronutrientes del Instituto de Nutrición y Tecnología de los Alimentos (INTA) de la Universidad de Chile entre Marzo y Julio del año 2011.

Criterios de exclusión: Mujeres con enfermedad aparente, excepto anemia; tabaquismo; embarazo e interés por embarazarse; lactancia; y consumo de micronutrientes en suplementos.

Consideraciones éticas: El presente estudio forma parte del Proyecto FONDECYT 1095038. El estudio fue aprobado por parte del Comité de Ética para Estudios en Humanos del Instituto de Nutrición y Tecnología de los Alimentos (INTA) de la Universidad de Chile. Antes del inicio del estudio se orientó a todas las participantes sobre los beneficios y posibles riesgos de participar en este estudio, así como también se obtuvo su consentimiento informado firmado.

Diseño de la intervención: En el día 1 se realizó una consejería nutricional presencial en grupo, la cual fue dirigida por un nutricionista investigador. El objetivo de la consejería fue informar a las participantes sobre los beneficios del consumo de alimentos fuentes de calcio y los efectos adversos de una baja ingesta dietaria de este mineral. Para la consejería nutricional se utilizó una presentación en multimedia, teniendo una duración de 45 minutos y se permitió un período de 30 minutos adicionales para preguntas y respuestas. En esta primera etapa, también se aplicó un cuestionario validado de Frecuencia de Consumo Semi Cuantitativo (CFCSC) resumido del último mes $(19,20)$ para determinar la ingesta basal de calcio. Adicionalmente, se estimó la ingesta de macronutrientes y de algunos micronutrientes, con la finalidad de detectar potenciales cambios en la adecuación dietaria ajenos a la modificación de adecuación dietaria específica de calcio.

Posteriormente, desde el mismo día 1, las participantes tuvieron acceso a un espacio virtual desarrollado a través de una aplicación Web2.0 disponible para redes sociales en donde se reforzaba la importancia de la ingesta de calcio dietario a través de consejería nutricional virtual. Además, se hizo un seguimiento de preguntas y respuestas relacionadas con el estudio. El acceso a este espacio virtual estuvo disponible para ser visto desde cualquier ordenador, ya sea en casa o en cualquier otra instalación o equipo que contara con acceso a internet. El seguimiento fue de dos meses, completándose nuevamente el CFCSC los días 30 y 62, habilitándose una encuesta virtual utilizando una aplicación de Google Drive. La encuesta virtual podía ser contestada desde los ordenadores dispuestos en el Instituto o a través de ordenadores en casa

\section{FIGURA 1}

Algoritmo para la cuantificación de ingesta diaria de macro y micronutrientes.

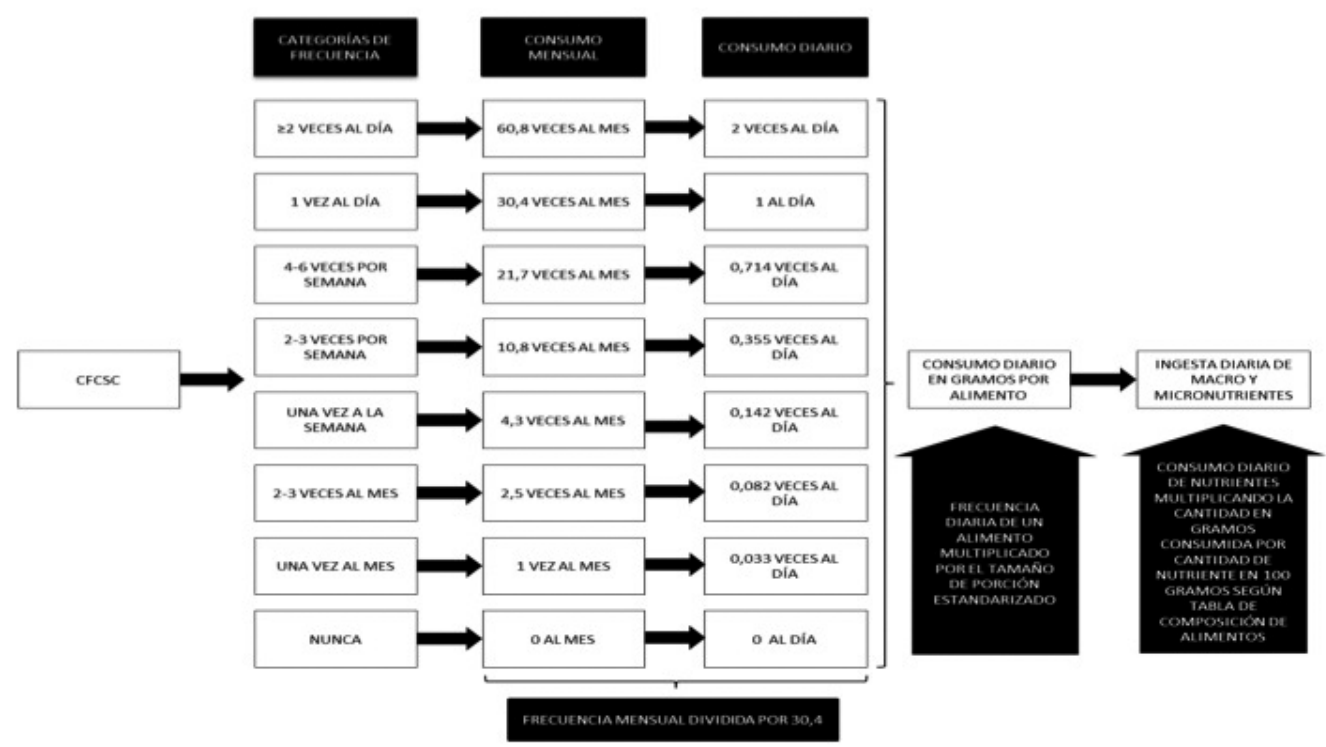

Adaptado de Pakseresht M, Sharma S, Cao X, Harris R, Caberto C, Wilkens LR, Hennis AJ, Wu SY, Nemesure B, Leske MC.

Validation of a quantitative FFQ for the Barbados National Cancer Study. Public Health Nutr 2010;14:426-34. 
u otro centro de informática accesible. Cuando la participante reportaba no tener acceso a internet, se citaba al Instituto para realizar la entrevista cara a cara con un nutricionista investigador.

Colección de los datos: El CFCSC del día 1 constaba de 27 secciones para evaluar la ingesta usual de alimentos y bebidas en los últimos 30 días, permitiendo registrar la frecuencia de consumo de alimentos específicos ya sea diaria, semanal, quincenal o mensual. El CFCSC constaba de ocho categorías de elección para frecuencia, desde nunca hasta $\geq 2$ veces al día (figura 1). Los tamaños de las porciones fueron estimadas utilizando tamaños estandarizados de medidas caseras de Chile, según Gattas y Aguayo 1977 (21). La ingesta de macronutrientes y minerales se calculó utilizando la Tabla de Composición Química de Alimentos de Chile (22).

La ingesta diaria de alimento fue determinada para cada sujeto. Las categorías de frecuencia de consumo en el CFCSC fueron convertidas a frecuencia mensual. La ingesta diaria de cada alimento en gramos fue obtenida dividiendo la ingesta mensual por 30,4 días (figura 1), según el método descrito por Pakseresh y col 2010 (20). La cantidad de macronutrientes y minerales para cada alimento ingerido, en sus respectivas unidades de medidas, fue obtenida multiplicando la cantidad en gramos ingerida por la cantidad de cada nutriente en 100 g según la Tabla de Composición Química de Alimentos de Chile (22). La ingesta total se obtuvo sumando la ingesta de cada nutriente por alimento evaluado. Se decidió analizar sólo la ingesta de macronutrientes (proteínas, carbohidratos y grasas) y de macro minerales (calcio, hierro, fósforo y sodio) debido a que podrían variar ante un cambio en el patrón de alimentación. Se omiten del análisis las vitaminas y elementos trazas en vista de que su requerimiento se cuantifican en unidades menores y representarían una dificultad para medir los cambios en la ingesta. Además de calcio se decidió medir otros macro minerales para estimar que no existiesen cambios ajenos a la intervención. Para la clasificación del porcentaje de adecuación de minerales se utilizó la referencia del Instituto de Medicina de los Estados Unidos (3), considerando adecuado un rango entre 90 y $110 \%$.

Indicadores antropométricos: El peso y la talla se midieron en el día 1 y el día 62 de la intervención a través de una balanza electrónica de precisión Hispana SECA (Model 700, Seca Mechanical Column Scales, Seca Corporation, Germany) con estadiómetro incluido y con una sensibilidad de 0,1 kg y $0,1 \mathrm{~cm}$, respectivamente. Para las mediciones antropométricas se tomaron tres lecturas y se promediaron los valores. Las participantes vistieron ropa ligera, no utilizaron calzado y mantuvieron la posición horizontal de Frankfurt. Con el peso y la talla se obtuvo el Índice de Masa Corporal (IMC), variable derivada de la relación entre el peso en kilogramos sobre la talla en metros al cuadrado $(\mathrm{Kg} / \mathrm{m} 2)$.

Análisis estadístico: El promedio \pm desviación estándar (DE) fue utilizado para presentar los datos continuos y frecuencia (porcentaje \%) para las variables categóricas. Un análisis de varianza de medidas repetidas (ANOVA) con una prueba de contraste de Bonferroni fue utilizado para determinar el cambio en la ingesta dietaria entre los tres CFCSC. Para comparar las características antropométricas al inicio y después de dos meses, se utilizó la prueba t de Student para medidas repetidas. Todos los análisis estadísticos fueron realizados con el programa STATA 11,0 (Stata Corp LP, College Station, Texas, USA). Se estableció la significancia estadística cuando el valor p de las pruebas fue menor de 0,05.

\section{RESULTADOS}

Se incluyeron en el protocolo inicial 28 mujeres (figura 2), finalizando el estudio 20 participantes. El motivo por el cual 8 participantes no completaron el estudio por no realizar las CFCSC en los días 30 y 62, principalmente por falta de acceso a la aplicación de internet, por no tener conexión a internet, no contar con un equipo computacional o por no contar con el conocimiento necesario para usar un computador, por no

\section{FIGURA 2}

Diagrama de flujo de los procesos a través de las fases del estudio.
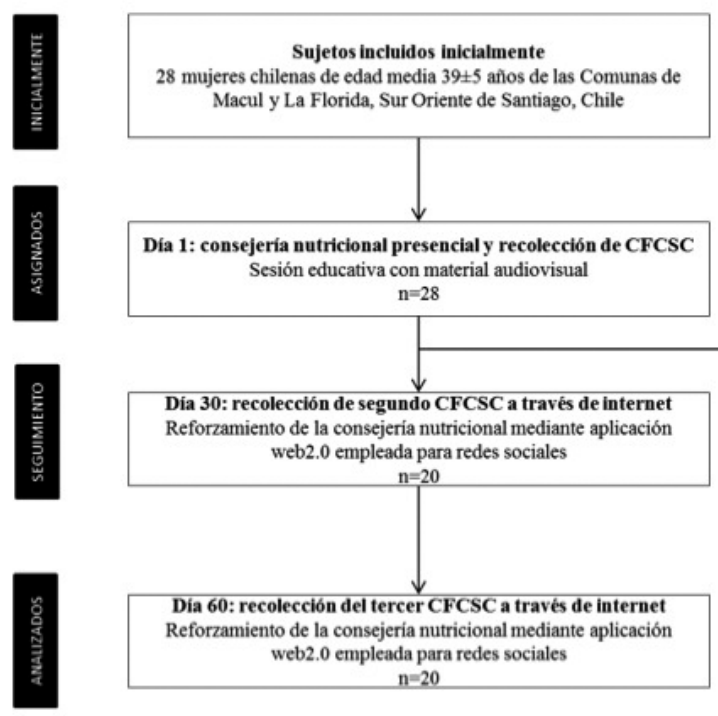

Dia 1: consejeria nutricional presencial y recolección de CFCSC Sesión educativa con material audiovisual $\mathrm{n}=28$

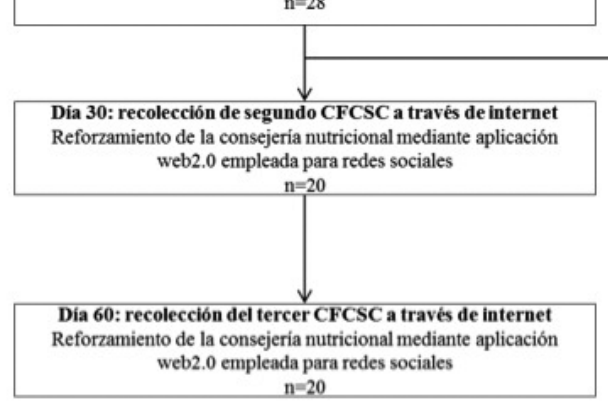

Se retiran 8 participantes

Reportan no completar el CFCSC a través de la internet 
ser posible la entrevista cara a cara, o por deserción. Sólo se incluyeron en el análisis final las 20 participantes que completaron todas las evaluaciones.

En cuanto a las características de las participantes, la edad promedio fue $39,3 \pm 4,5$ años. La talla promedio fue 1,56 $\pm 0,07 \mathrm{~m}$. Luego de tres meses de intervención, no hubo cambios significativos en las medidas antropométricas de las participantes; el peso promedio inicial y final fue $62,3 \pm 6,7$ y $62,6 \pm 6,7 \mathrm{~kg}$, respectivamente $(p=0,84)$. El IMC promedio inicial y final fue $25,7 \pm 3,2$ y $25,8 \pm 3,0 \mathrm{~kg} / \mathrm{m} 2$, respectivamente $(p=0,89)$ (tabla 1$)$.

La estimación de ingesta dietaria diaria de calcio aumentó progresivamente después de dos meses de consejería virtual y presencial (ANOVA, medidas repetidas; $F=4,43 ; p=0,02$ ). La ingesta dietaria de calcio en el período basal fue de 308 $\pm 116 \mathrm{mg} / \mathrm{d}$, al día 30 fue $354 \pm 138 \mathrm{mg} / \mathrm{d}$ y al día 62 fue $412 \pm 188$ (tabla 2). La estimación de ingesta energética, de macronutrientes y minerales a excepción de calcio permaneció constante entre la primera medición y después de dos meses de seguimiento.

Aunque la estimación de ingesta dietaria de calcio presumiblemente aumentó por efecto de la consejería virtual y presencial, el 95\% de las participantes no logró cubrir el porcentaje de adecuación esperado al final de la intervención, la media del porcentaje de adecuación (PA) final para calcio fue $68 \pm 19 \%$ (tabla 3).

\section{DISCUSIÓN}

El presente estudio encontró un aumento significativo en las ingesta estimada de calcio en mujeres en edad fértil del Sur Oriente de Santiago de Chile. Sin embargo, aunque la consejería aumento la ingesta de calcio, esta no fue suficiente para cubrir los requerimientos, siendo bajo el 50\% del EAR esperado (1000 mg/día) en las tres mediciones realizadas (3).

La consejería nutricional sería una intervención a considerar para mejorar los conocimientos y la adherencia a óptimos patrones alimentarios de la población. Existe evidencia de que la consejería nutricional puede promover una dieta saludable así como mejorar el comportamiento dietético, incluyendo la reducción de las grasas saturadas y el aumento de las ingesta de frutas y vegetales $(23,24)$. Varios estudios han reportado el efecto de la consejería nutricional sobre el incremento en la ingesta de calcio $(25,26)$. En nuestro estudio el incremento observado, respecto al día 1 fue (delta) $47 \pm 94 \mathrm{mg} / \mathrm{d}$ al día

\section{TABLA 1}

Características antropométricas de las mujeres del estudio

\begin{tabular}{|c|c|c|c|c|}
\hline Variables $(n=20)$ & Día 1 & Día 62 & $\mathrm{~T}^{\mathrm{a}}$ & $\mathrm{p}$ \\
\hline Talla (m) & $1,56 \pm 0,07$ & - & - & - \\
\hline Edad (años) & $39,3 \pm 4,5$ & - & - & - \\
\hline Peso (Kg) & $62,3 \pm 6,7$ & $62,6 \pm 6,7$ & $-0,20$ & 0,84 \\
\hline$I M C$ & $25,6 \pm 3,2$ & $25,8 \pm 3,0$ & $-0,14$ & 0,89 \\
\hline
\end{tabular}

${ }^{1}$ Valores presentados como media \pm desviación estándar

${ }^{a}$ Valor de la prueba $t$ de Student para muestras pareadas a dos colas

IMC= índice de masa corporal

\section{TABLA 2}

Ingesta de energía, macro y micronutrientes en las mujeres del estudio.

\begin{tabular}{|c|c|c|c|c|c|}
\hline Consumo día & Evaluación 1 & Evaluación 2 & Evaluación 3 & $\mathrm{~F}$ & $\mathrm{p}$ \\
\hline Energía (Kcal/d) & $1877 \pm 287$ & $1989 \pm 381$ & $1982 \pm 360$ & 1,11 & 0,34 \\
\hline Carbohidratos (g/d) & $209 \pm 40$ & $219 \pm 42$ & $222 \pm 52$ & 0,74 & 0,48 \\
\hline Proteínas (g/d) & $57 \pm 15$ & $64 \pm 21$ & $61 \pm 16$ & 1,16 & 0,32 \\
\hline Grasas (g/d) & $90 \pm 12$ & $95 \pm 26$ & $95 \pm 17$ & 0,77 & 0,47 \\
\hline Fibra (mg/d) & $4 \pm 2$ & $3 \pm 2$ & $4 \pm 3$ & 1,66 & 0,20 \\
\hline Hierro (mg/d) & $11,1 \pm 2,9$ & $12,4 \pm 3,1$ & $12,0 \pm 3,2$ & 1,70 & 0,20 \\
\hline${ }^{1}$ Calcio $(\mathrm{mg} / \mathrm{d})^{+}$ & $308 \pm 116 a$ & $354 \pm 138 b$ & $412 \pm 188 c$ & 4.43 & 0.02 \\
\hline $\begin{array}{l}\text { Fósforo }(\mathrm{mg} / \mathrm{d}) \\
\text { Sodio }(\mathrm{mg} / \mathrm{d})\end{array}$ & $\begin{array}{c}720 \pm 160 \\
1298 \pm 398\end{array}$ & $\begin{array}{c}803 \pm 234 \\
1329 \pm 492\end{array}$ & $\begin{array}{c}817 \pm 220 \\
1352 \pm 376\end{array}$ & $\begin{array}{l}2,22 \\
0,25\end{array}$ & $\begin{array}{l}0,12 \\
0,78\end{array}$ \\
\hline \multicolumn{6}{|c|}{$\begin{array}{l}\text { Valores presentados como promedios } \pm \text { desviación estándar. } \\
\text { 'Ingesta de calcio dietario } \\
\text { 'ANOVA medidas repetidas; Bonferroni, test de comparación múltiple (evaluación } 3 \text { vs evaluación } 1 \text { p<0.05). } \\
\text { CFC Evaluación } 1=\text { tiempo basal; Evaluación } 2=1 \text { mes; Evaluación } 3=2 \text { meses } \\
\text { Kcal= kilocalorías; g= gramos; } \mathrm{mg}=\text { miligramos }\end{array}$} \\
\hline
\end{tabular}


30 y $103 \pm 169 \mathrm{mg} / \mathrm{d}$ al día 62 .

En el presente estudio se observó que la consejería nutricional realizada virtualmente podría ser útil en incrementar las ingesta de calcio dietario diario. Similar a nuestro hallazgo, otros autores han evaluado los efectos que tiene sobre el consumidor el uso de internet para tomar decisiones, mejorar conocimientos, actitudes y utilización de la información sobre temas de salud, reportándose efectos positivos en los programas de educación nutricional y pérdida de peso (17). Por su parte, Asakawa y cols (2011), en un estudio en Japón que incluyó 182 mujeres saludables de 18 a 25 años de edad con un seguimiento de 6 meses, en el cual se buscaba determinar si la intervención nutricional a través del internet era capaz de incrementar la resistencia ósea, observó un aumento en el promedio de la ingesta dietaria diaria de calcio, siendo el cambio respecto al período basal de 216,3 \pm 85,9 mg/día adicionales en el grupo intervenido (27).

Desde un punto de vista preventivo, sería recomendable promocionar un aumento de la ingesta dietaria de calcio a toda la población, mediante campañas de educación pública utilizando estrategias de comunicación social masiva tradicionalmente empleadas en la publicidad comercial, así como también, promoción del consumo de alimentos ricos en calcio (tabla 4) con bajo contenido de grasas, a fin de cumplir con la EAR para este mineral.

En Chile, consistente con nuestro resultados, Aguirre y cols (2007) en un estudio en mujeres obesas reportaron una ingesta inferior al $50 \%$ de las EAR, tanto al inicio como al final de la intervención $(540 \pm 287$ y $480 \pm 209 \mathrm{mg} / \mathrm{d}$, respectivamente) (12). De igual manera, Castillo y cols (1997), en otro estudio sobre patrones alimentarios en la Región Metropolitana de Chile, observaron que la ingesta de calcio estaba bajo el 50\% en adultos que asisten a centros de atención primaria (11). Esta tendencia deficitaria de ingesta de calcio se observó también en grupos vulnerables, como las adolescentes en período de lactancia $(10,28)$. En general, varios estudios demuestran una baja ingesta de calcio en las mujeres chilenas $(11,12)$.

El bajo consumo de calcio en la dieta, inclusive entre aquellas mujeres en estados fisiológicos comprometidos (embarazo y lactancia), está muy relacionado a sus conocimientos y creencias propias. Se ha descrito que el número de hijos, la puntuación de la autopercepción de la salud, el IMC, el nivel de educación, y las experiencias con exámenes relacionados a la salud ósea e historia familiar explican en su conjunto 31,8\% de la variación de la ingesta de calcio (29). Otros factores relacionados al bajo consumo de calcio y su biodisponibilidad están ligados a las actitudes y pensamientos negativos hacia el calcio y al consumo de café (30).

En Chile, otro elemento importante a analizar respecto a la ingesta de calcio es la dureza del agua, ya que este factor aumenta la estimación de la ingesta de calcio en la dieta. El contenido promedio de calcio en el agua de la región Sur Oriente de Santiago es de 133,91 mg/L (31). Al considerar en nuestro estudio el aporte de calcio de una ingesta promedio de agua de aproximadamente $2 \mathrm{~L} /$ día, la ingesta diaria de calcio al día 62 aumentaría de 412 a 679 mg/d. El contenido de calcio en el agua es variable y depende de la naturaleza geológica del área donde se asienta el acuífero y su asociación con cuencas de captación de rocas sedimentadas. La dureza del agua se debe a cationes metálicos divalentes, entre ellos el calcio, que se combinan con aniones. Sin embargo, es necesario realizar un análisis más profundo de la relación entre la ingesta de calcio y el consumo real de agua en esta población.

Chile ha experimentado una rápida transición nutricional durante los últimos años, caracterizada por una disminución de la tasa de desnutrición primaria e incidencia de mortalidad materno-infantil. Sin embargo, estos cambios han sido acompañados por un dramático aumento en la prevalencia de sobrepeso y obesidad, relacionado con una alta ingesta de grasas, azúcares simples y sodio, y bajos niveles de actividad física, coexistiendo con deficiencias de micronutrientes (32). En ese sentido, nuestros resultados indican que hay un consumo elevado de energía y un bajo consumo de micronutrientes, en particular de calcio. Recientemente se ha relacionado la ingesta adecuada de calcio con la prevención de exceso de adiposidad corporal, hipertensión arterial

\section{TABLA 3}

Adecuación macronutrientes y macro minerales de la ingesta al final (día 62) en mujeres de 35-45 años de edad.

\begin{tabular}{|c|c|c|c|c|c|c|}
\hline & \multirow[t]{2}{*}{$\mathrm{EAR}^{1}$} & \multirow{2}{*}{$\begin{array}{l}\text { media } \pm \text { DE } \\
\text { de ingesta }\end{array}$} & \multirow{2}{*}{$\begin{array}{c}\text { media } \pm \text { DE de } \\
\operatorname{PA}^{2}(\%)\end{array}$} & \multicolumn{3}{|c|}{ N(\%) de sujestos según adecuación } \\
\hline & & & & $<90 \%)$ & $\begin{array}{l}\text { Adecuado } \\
\text { (90-110\%) }\end{array}$ & $>110 \%$ \\
\hline Calorías cal/d & $1600-1700$ & $1982 \pm 360$ & $120 \pm 22$ & $2(10 \%)$ & $6(30 \%)$ & $12(60 \%)$ \\
\hline Proteina g/d & 46 & $61 \pm 16$ & $133 \pm 35$ & - & $6(30 \%)$ & $14(70 \%)$ \\
\hline Carbohidratos g/d & 130 & $222 \pm 52$ & $171 \pm 40$ & - & $1(5 \%)$ & $19(95 \%)$ \\
\hline Grasas g/d & 53 & $95 \pm 17$ & $179 \pm 33$ & - & - & $20(100 \%)$ \\
\hline Fibra g/d & 25 & $4 \pm 3$ & $17 \pm 12$ & $20(100 \%)$ & - & - \\
\hline${ }^{3}$ Calcio mg/d & 1000 & $677 \pm 205$ & $68 \pm 19$ & $1(5 \%)$ & $1(5 \%)$ & $18(90 \%)$ \\
\hline Hierro mg/d & 18 & $12 \pm 3$ & $67 \pm 18$ & $18(90 \%)$ & $2(10 \%)$ & - \\
\hline Fósforo mg/d & 700 & $834 \pm 235$ & $117 \pm 32$ & $3(15 \%)$ & $7(35 \%)$ & $10(50 \%)$ \\
\hline Sodio $\mathrm{mg} / \mathrm{d}$ & 1500 & $1000 \pm 380$ & $90 \pm 25$ & $8(40 \%)$ & $7(35 \%)$ & $5(25 \%)$ \\
\hline $\begin{array}{l}{ }^{1} E A R: \text { promedio requerid } \\
\text { 2PA: porcentaje de adecu }\end{array}$ & do & . & 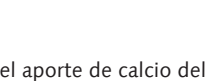 & Chile. & & \\
\hline
\end{tabular}


(HTA), enfermedad cardiovascular $(\mathrm{ECV})$ e resistencia a la insulina $(33,34)$.

Como fortalezas del presente estudio se puede mencionar que se hicieron mediciones en tiempos diferentes y se logró obtener repetibilidad de los datos observados en el resto de variables evaluadas, enfatizando la idea de que los cambios encontrados en la ingesta de calcio fueron efecto de la consejería nutricional impartida (presencial y virtual). De la misma manera, nuestro hallazgo podría validar la aplicación del CFCSC a través de internet. Igualmente, si bien se ha recomendado la técnica por recordatorio de 24 horas para medir ingesta dietaría, ésta metodología tiene la desventaja de que sólo mide ingesta de un día, o por su complejidad, de un corto período de tiempo. Por su parte, el CFCSC nos permite un estimado de la ingesta del último mes, siendo el método más adecuado para este tipo de análisis.

El análisis presentado en el este artículo corresponde a un objetivo secundario de un estudio diseñado con el propósito de evaluar la absorción de hierro en presencia de calcio. Sin embargo, representa una oportunidad de evaluar el efecto de la consejería sobre el consumo de alimentos con alto contenido de calcio. El diseño del estudio no contó con un grupo control. La ausencia de grupo control debilita el suponer que el aumento observado de alimentos con alto contenido de

\section{TABLA 4}

Alimentos chilenos seleccionados con alto contenido de calcio.

\section{Alimento}

Leche en polvo $26 \%$ grasa

Leche en polvo $12 \%$ grasa

Leche en polvo descremada

Leche evaporada

Leche de vaca pasteurizada

Yogurt natural

Yogurt batido simple

Yogurt con sabor

Yogurt con frutas

Yogurt dieta

Queso gouda

Queso cabra

Quesillo

Leche Purita

Leche Purita Cereal

Leche Purita Modificada

Sardina en aceite

Sardina en salsa

Choritos

Pejerrey

Macha

Acelga cruda

Brócoli

Acelga cocida

Aceituna

Frejol cocido

Soya

Frejol crudo

Haba seca

Garbanzo crudo

Poroto granado

Garbanzo cocido

Almendra

Higos

Avena

Semola

Maní salado
Calcio (mg) en $100 \mathrm{~g}$

1230

1020

1020

231

123

150

145

127

105

117

1076

626

487

912

500

500

374

262

202

147

87

124

103

101

87

260

197

164

141

134

96

78

294

180

148

117

105

Fuente: Tabla de Composición Química de Alimentos en Chile. 
calcio es atribuible a la consejería virtual y presencial. Factores socioeconómicos pueden influenciar cambios en la ingesta dietética. Por otro lado, no se evaluó el estado nutricional de calcio a través de determinaciones bioquímicas. Lo anterior, implica que los cambios dietarios no necesariamente implican mejorías en el estado nutricional de este mineral. Aun considerando estas limitaciones, nuestro estudio muestra un alto porcentaje de inadecuación dietaria en la línea de base y al final del estudio. Existiendo un aumento en la ingesta de alimentos con alto contenido de calcio no se lograría una adecuación dietaria al efectuar la consejería. Sin embargo, es importante reconocer que existen grandes dificultades en traducir el reporte de ingesta dietaria a su aporte diario, dado que las tablas de composición química de alimentos en general se desarrollan en base a otros alimentos, dada las diferencias en biodisponibilidad de los nutrientes.

La realización de la estimación de la ingesta de manera virtual podría presentar ciertas desventajas, en particular considerando aquellas mujeres sin acceso a este tipo de tecnología. En nuestro estudio, las encuestas se aplicaron al día 30 y 62, a través de una aplicación Google Drive, resultando en que ocho de las 28 participantes no pudieran completar el estudio debido a que no contaban con acceso a internet, ya sea por falta de equipo o por conocimientos de cómo utilizarlo; así también, limitaba a estas participantes de reforzar y/o preguntar vía internet a través del grupo en redes sociales. Por lo tanto, los resultados sólo podrían ser representativos de personas con acceso a tecnologías de información y comunicación. De igual manera, coincidentemente el nivel socioeconómico bajo podría presentar una ingesta de calcio menor. El sesgo del recuerdo también puede ser considerado como una limitante debido a que el CFCSC depende de la memoria.

Por último, pudiera ser considerada también como una limitante más del estudio el hecho de que no se logró aumentar la ingesta de calcio a una significancia clínica o al menos a más del 50\% de la ingesta recomendada de 1000 mg/d. Por otro lado, se podría considerar que el CFCSC como método de estimación de la ingesta sobreestima el valor de la misma (35). Esto puede deberse a que el CFCSC permite el reporte de alimentos que no son ingeridos con regularidad por la población estudiada $(35,36)$. Además, el tamaño de la porción, que al no ser adecuada a la población en estudio, podría conducir a la inexactitud en la cuantificación de la ingesta (37).

Conclusión: La consejería nutricional podría aumentar la ingesta de alimentos fuentes de calcio en mujeres en edad fértil chilenas. No obstante, la ingesta dietaria estimada de calcio al final de la intervención no alcanzó más del 50\% de los requerimientos.

\section{RESUMEN}

El calcio juega un rol crucial en la regulación de procesos metabólicos, especialmente en la función neuromuscular y en la salud ósea. Sin embargo, la ingesta dietaria de calcio en mujeres en edad fértil de Chile no cubre los requerimientos. Objetivo: determinar sí la consejería nutricional presencial y virtual incrementa la ingesta dietaria de calcio en mujeres chilenas de edad fértil. Sujetos y métodos: la ingesta dietaria de calcio en 20 mujeres (edad media de $39 \pm 5$ años) antes y después de una intervención de educación alimentaria y nutricional fue comparada. La consejería nutricional fue impartida para promover el consumo diario de alimentos ricos en calcio. Además, se habilitó un espacio virtual para reforzar la consejería nutricional. En el día 1, 30 y 62 se aplicaron cuestionarios de frecuencia de consumo semi-cuantitativos.
La ingesta dietaria de calcio incrementó significativamente (ANOVA $F=4.43, P=0.02$ ) después de la intervención. La ingesta dietaria de calcio fue $308 \pm 116 \mathrm{mg} / \mathrm{d}$ al día 1, $354 \pm$ $138 \mathrm{mg} / \mathrm{d}$ al día 30, e incrementó a $412 \pm 188 \mathrm{mg} / \mathrm{d}$ después de 62 días. Al final de la intervención, el 95\% de las participantes no cubrieron sus requerimientos diarios de calcio; la media del porcentaje de adecuación para la ingesta de calcio fue $68 \pm 19 \%$. La consejería nutricional podría incrementar la ingesta dietaria de calcio en mujeres de edad media en Chile. Sin embargo, el aumento no es suficiente para cubrir los requerimientos de calcio.

Palabras clave: calcio, micronutrientes, ingesta dietaria, requerimientos nutricionales, consejería nutricional.

Agradecimientos: Los autores agradecen a las participantes del estudio por su colaboración, así como también a las Nutricionistas Paulina Castiglioni por su apoyo en la recolección y codificación de los datos alimentarios y antropométricos. El estudio fue financiado por el Proyecto FONDECYT 1095038, Santiago, Chile.

\section{BIBLIOGRAFÍA}

1. Weaver CM. Calcio. In: Bowman BA, Russel RM, editors. Conocimientos Actuales sobre Nutrición. Washington DC: Organización Panamericana de la Salud; 2003. p. 873.

2. Heaney RP. Calcium intake and disease prevention. Arq Bras Endocrinol Metabol. 2006;50(4):685-93.

3. Institute of Medicine. Dietary Reference Intakes for Calcium and Vitamin D. Dietary Reference Intakes for Calcium and Vitamin D. Washington DC: The National Academies Press; 2011.

4. Heaney RP. Dairy and bone health. J Am Coll Nutr. 2009;28 Suppl 1:82S-90S.

5. Goulding A. Risk factors for fractures in normally active children and adolescents. Med Sport Sci. 2007;51:102-20.

6. Henry YM, Fatayerji D, Eastell R. Attainment of peak bone mass at the lumbar spine, femoral neck and radius in men and women: relative contributions of bone size and volumetric bone mineral density. Osteoporos Int. 2004;15(4):263-73.

7. Frenkel B, Hong A, Baniwal SK, Coetzee GA, Ohlsson C, Khalid $O$, et al. Regulation of adult bone turnover by sex steroids. J Cell Physiol. 2010;224(2):305-10.

8. World Health Organization. Scientific group on the assessment of osteoporosis at primary health care level. Summary Meeting Report. Brussels, Belgium, 5-7 May 2004. Geneva, Switzerland; 2007 p. 13.

9. Monge-Rojas $R$, Nuñez HP. Dietary calcium intake by a group of 13 18-year-old Costa Rican teenagers. Arch Latinoam Nutr. 2001;51(2):127-31.

10. Fernández-Ortega M. Food calcium intake in teenager women in Panama. Arch Latinoam Nutr. 2008; 58(3): 286-91.

11. Castillo C, Atalah E, Benavides X, Urteaga C. Food patterns among adults visiting primary care clinics in the metropolitan region. Rev Med Chil. 1997;125(3):283-9.

12. Aguirre MEO, Ruz M, Carrasco FN, Rebolledo AA, Araya $K$, Codoceo JA, et al. Efecto de dietas con restricción moderada de energía sobre el estado nutricional de algunos minerales en mujeres obesas. Arch Latinoam Nutr. 2007; 57(3):238-47.

13. Pimentel GD, Arimura ST, de Moura BM, Silva MER, de Sousa M V. Short-term nutritional counseling reduces body mass index, waist circumference, triceps skinfold and 
triglycerides in women with metabolic syndrome. Diabetol Metab Syndr. 2010;2(2):13.

14. Stark LJ, Janicke DM, McGrath AM, Mackner LM, Hommel $K$ a, Lovell D. Prevention of osteoporosis: a randomized clinical trial to increase calcium intake in children with juvenile rheumatoid arthritis. J Pediatr Psychol. 2002; 30(5):377-86.

15. Celio AA, Winzelberg AJ, Wilfley DE, Eppstein-Herald D, Springer EA, Dev $P$, et al. Reducing risk factors for eating disorders: comparison of an Internet- and a classroomdelivered psychoeducational program. J Consult Clin Psychol. 2000;68(4):650-7.

16. Winzelberg AJ, Eppstein D, Eldredge $K L$, Wilfley D, Dasmahapatra $R, \operatorname{Dev} P$, et al. Effectiveness of an Internet-based program for reducing risk factors for eating disorders. $J$ Consult Clin Psychol. 2000;68(2):346-50.

17. Bessell TL, MCDonald S, Silagy CA, Anderson JN, Hiller JE, Sansom LN. Do Internet interventions for consumers cause more harm than good? A systematic review. Heal Expect. 2002;5(1):28-37.

18. Ríos-Castillo I, Olivares M, Brito A, López de Romaña D, Pizarro F. One-month of calcium supplementation does not affect iron bioavailability: A randomized controlled trial. Nutrition. 2014;30(1):44-8.

19. Olivares M, Pizarro F, De Pablo S, Araya M, Uauy R. Iron, Zinc, and Copper: Contents in Common Chilean Foods and Daily Intakes in Santiago, Chile. Nutrition. 2004. p. 205-12.

20. Pakseresht $M$, Sharma S, Cao X, Harris $R$, Caberto $C$, Wilkens $L R$, et al. Validation of a quantitative FFQ for the Barbados National Cancer Study. Public Health Nutr. 2011. p. 426-34.

21. Gattás V, Aguayo M. Tabla de pesos y medidas prácticas de alimentos, su equivalencia en gramos y aporte nutritivo. Santiago, Chile: Universidad de Chile, Instituto de Nutrición y Tecnología de los Alimentos; 1977.

22. Schmidt-Hebbel H, Pennacchiotti I, Masson L, Mella MA. Tabla de Composición Química de Alimentos Chilenos. Santiago, Chile: FAO y Ministerio de Salud de Chile; 1992.

23. Castillo C Á, Smith $G C$, Hirsch B S, Brito O A. ¿Es efectiva la consejería para aumentar el consumo de frutas y verduras y disminuir el riesgo cardiovascular en prevención secundaria?: una revisión. Rev Chil Nutr. 2008; 35(2):123-9.

24. Pignone MP, Ammerman A, Fernandez L, Orleans CT, Pender $N$, Woolf S, et al. Counseling to promote a healthy diet in adults: A summary of the evidence for the U.S. Preventive Services Task Force. Am J Prev Med. 2003;24(1):75-92.

25. Wong SY, Lau EM, Lau WW, Lynn HS. Is dietary counselling effective in increasing dietary calcium, protein and energy intake in patients with osteoporotic fractures?
A randomized controlled clinical trial. J Hum Nutr Diet. 2004;17(4):359-64.

26. Hien VTT, Khan NC, Mai LB, Lam NT, Phuong TM, Nhung $B T$, et al. Effect of community-based nutrition education intervention on calcium intake and bone mass in postmenopausal Vietnamese women. Public Health Nutr. 2009;12(5):674-9.

27. Kazumi A. Effect of educational intervention using the Internet on quantitative ultrasound parameters in prevention of osteoporosis: a randomized controlled trial in young Japanese women. Int J Womens Health. 2011;3:415-22.

28. Goldberg GR, Jarjou LMA, Cole TJ, Prentice A. Randomized, placebo-controlled, calcium supplementation trial in pregnant Gambian women accustomed to a low calcium intake: effects on maternal blood pressure and infant growth. Am J Clin Nutr. 2013;98(4):972-82.

29. Chang S-F. A cross-sectional survey of calcium intake in relation to knowledge of osteoporosis and beliefs in young adult women. Int J Nurs Pract. 2006;12(1):21-7.

30. Lagos Ruiz MJ, Montenegro Castillo YR, Niño Orbegoso GP, Barrera Perdomo M del P. Conocimientos, actitudes, prácticas y consumo de calcio en un grupo de mujeres adultas, Bogotá, 2003-2004. Rev Cuba Salud Pública. 2005;31(3):211-6.

31. Neira Gutierrez MA. Dureza en aguas de consumo humano y uso industrial, impactos y medidas de mitigación. Estudio de caso: Chile. Universidad de Chile; 2006. p. 95.

32. Garmendia ML, Corvalan C, Uauy R. Addressing malnutrition while avoiding obesity: minding the balance. Eur J Clin Nutr. Nature Publishing Group; 2013;67(5):513-7.

33. Martini LA, Catania AS, Ferreira SRG. Role of vitamins and minerals in prevention and management of type 2 diabetes mellitus. Nutr Rev. 2010;68(6):341-54.

34. Barba G, Russo P. Dairy foods, dietary calcium and obesity: A short review of the evidence. Nutr Metab Cardiovasc Dis. 2006;16(6):445-51.

35. Fumagalli F, Pontes Monteiro J, Sartorelli DS, Vieira MNCM, de Lourdes Pires Bianchi M. Validation of a food frequency questionnaire for assessing dietary nutrients in Brazilian children 5 to 10 years of age. Nutrition. 2008;24(5):427-32.

36. Kowalkowska J, Slowinska M a, Slowinski D, Dlugosz A, Niedzwiedzka $E$, Wadolowska L. Comparison of a full foodfrequency questionnaire with the three-day unweighted food records in young Polish adult women: implications for dietary assessment. Nutrients. 2013;5(7):2747-76.

37. 37.Wengreen HJ, Munger RG, Wong SS, West NA, Cutler R. Comparison of a picture-sort food-frequency questionnaire with 24-hour dietary recalls in an elderly Utah population. Public Health Nutr. 2001;4(5):961-70. 